\title{
PEROXY COMPOUNDS AS ECO-FRIENDLY CATALYSTS FOR NITRATION OF AROMATIC COMPOUNDS USING SODIUM NITRITE IN AQUEOUS BISULFATE MEDIUM
}

\author{
Muppidi Suresh $^{1,2}$, M. Venkateswarlu, K. C. Rajanna ${ }^{1, *}$ and P. K. Saiprakash ${ }^{1}$ \\ ${ }^{1}$ Department of Chemistry, Osmania University, Hyderabad-500 007, T. S (India) \\ ${ }^{2}$ Department of Chemistry, Telangana University, Dichpally, Nizambad-503322, T. S (India) \\ *E-mail:kcrajannaou@yahoo.com
}

\begin{abstract}
Peroxy compounds such as $\mathrm{H}_{2} \mathrm{O}_{2}$, peroxydisulfate (PDS), peroxymonosulfate (PMS), sodium perborate (SPB), and tert-butyl hydroperoxide (TBHP) have been used as efficient catalysts for nitration of aromatic and heteroaromatic compounds in presence of $\mathrm{NaNO}_{2} / \mathrm{KHSO}_{4}$ under mineral acid-free conditions. The reactions afforded very good yields of under different conditions. Depending on the nature of peroxide and substrate, reaction times recorded under conventional conditions reduced from ( 2 to $7 \mathrm{hrs}$ ) hours to (25 to 60) minutes with grindstone technique, which in turn reduced to (25 to 120$)$ seconds in microwave assisted reactions. Product yields are also increased from 6 to $10 \%$ under microwave irradiation as compared to conventional procedures. All synthesized compounds were characterized by ${ }^{1} \mathrm{H}$ NMR and mass spectral studies.
\end{abstract}

Keywords: $\mathrm{H}_{2} \mathrm{O}_{2}$, peroxydisulfate, peroxymonosulfate, sodium perborate, tert-butyl hydroperoxide; nitration; Grindstone technique; microwave irradiation; rate accelerations.

(C) RASĀYAN. All rights reserved

\section{INTRODUCTION}

Nitration of aromatic and heteroaromatic compounds occupies one of the most important and forefront positions in the fields of electrophilic aromatic substitution reactions. Large numbers of nitroarenes are widely used as important intermediates and precursors during the synthesis of organic and organometallic compounds, pharmaceuticals, explosives, dyes, polymers, pesticides, plastics, drugs, and medicines, which make nitration of aromatic compounds as an area of evergreen interest to synthetic chemists. ${ }^{1-3}$ The most popular and oldest classical method of nitration is the mixed acid or an acid mixture method (mixture of two strong acids, sulfuric and nitric acid) which is still popular and in use. ${ }^{1}$ However, few nitration protocols also involved the use of nitric acid diluted in water or acetic acid. But the classical method of nitration is a notoriously polluting process because it generates large quantities of hazardous waste acid streams. These outlets are root causes of the environmental pollution. Besides, this separation of the nitro products from the acid is often a difficult and energy consuming process that implies a basic aqueous work-up. To overcome these issues, several modified protocols have been developed over the years. ${ }^{3-20}$ One of the forefront reagents developed in direction is nitronium tetrafluoroborate, $\left[\left(\mathrm{NO}_{2}\right)^{+}\left(\mathrm{BF}_{4}\right)^{-}\right]$, which has several advantages over nitric acid, apart from supplementing isolated the active nitronium ion ingredient. ${ }^{3}$ There are several other protocols that avoided the use of sulfuric acid completely, but used small amounts of $\mathrm{HNO}_{3}$ along with other co-catalysts to accomplish nitration. ${ }^{4-14}$ Micelle-forming surfactants, ${ }_{15-17}$ supported catalysts such as $\mathrm{P}_{2} \mathrm{O}_{5}$ immobilized on silica gel, ${ }^{18}$ metalmodified montmorillonite, ${ }^{19} \mathrm{Al}\left(\mathrm{H}_{2} \mathrm{PO}_{4}\right)_{3}$ (ref.20), and $\mathrm{V}(\mathrm{V})$ heteropolyacids ${ }^{21}$ have also been used as efficient catalysts to synthesize nitroaromatic compounds in the presence of dilute $\mathrm{HNO}_{3}$. Literature reports also revealed the use of an array of metal nitrates ${ }^{22-31}$ and melamine nitrate ${ }^{32}$ were also used as effective nitrating agents for the synthesis of nitro-organic compounds. Ipso nitration of aryl boronic acids was reported ${ }^{33}$ in aqueous media using $\mathrm{NaNO}_{2} / \mathrm{Cu}_{2} \mathrm{O} / \mathrm{aqNH}_{3}$. Few acid-free nitration protocols ${ }^{34-42}$ were also developed using $\mathrm{NH}_{4} \mathrm{NO}_{3} / \mathrm{KHSO}_{4}, \mathrm{~N}_{2} \mathrm{O}_{5}$ and $\mathrm{ClNO}_{2}$, TBAD and TBAC with $\mathrm{NaNO}_{2}$, quaternary ammonium nitrate, $\mathrm{N}_{2} \mathrm{O}_{5} / \mathrm{SO}_{2}$, tertiary butyl nitrite, $\mathrm{MeNO}_{2}$ with $\mathrm{K}_{2} \mathrm{CO}_{3} / \mathrm{Al}_{2} \mathrm{O}_{3}$. The efforts of 
Paul Anastas and Warner ${ }^{43-45}$ to overcome several environmental and cost-provoking issues lead to the development of twelve points of green chemistry, which served as twelve commandments for the chemists and biochemists to design and propagate several environmentally safe and economically viable synthetic protocols. The use of solvent-free methods such as mortar-pestle ${ }^{46-49}$, and microwave irradiation ${ }^{50-53}$ techniques made a landmark in organic synthesis because these methods are not only solvent-free but also operationally simple and satisfy both economic and environmental demands by replacing the toxic solvents. These methods are also associated with ease of manipulation and in several cases enhanced selectivity. On the other hand, in the recent past, potassium bisulfate $\left(\mathrm{KHSO}_{4}\right)$ is explored as an eco-friendly, inexpensive, non-toxic, and easy available efficient green catalyst for various organic transformations under homogeneous and heterogeneous conditions. ${ }^{38,39}$ A review article published by Baghernejad ${ }^{39}$ provides an excellent bibliography depicting an array of synthetic strategies in which $\mathrm{KHSO}_{4}$ is used as a catalyst. In many cases, the catalyst was recovered, activated and reused for three to four consecutive times with only slight variation in the yields of the products. Encouraged by the striking catalytic applications of $\mathrm{KHSO}_{4}$, we have developed certain nitration protocols using $\mathrm{NaNO}_{2} /$ peroxy compounds for the synthesis of nitroarenes using small (millimolar) amounts of $\mathrm{KHSO}_{4}$. The peroxy compounds such as $\mathrm{H}_{2} \mathrm{O}_{2}$, tertbutyl hydroperoxide (TBHP), Potassium peroxydisulfate $\left(\mathrm{K}_{2} \mathrm{~S}_{2} \mathrm{O}_{8}\right.$ or PDS), peroxymonosulfate (oxone or PMS), and sodium perborate (SPB) are used as catalysts in this study are laboratory benchtop chemicals and are environmentally safe and economically viable.

\section{EXPERIMENTAL}

The chemicals used in this study were procured from Avra, Aldrich, Arcos Organics or SD -fine chemicals. BUCHI B-545 capillary melting point apparatus was used to determine the melting points of the compounds. Bench mate model microwave oven (CEM-908010, 300W) was used to perform microwave assisted experiments in this study. Thin layer chromatography (TLC) experiments were carried out using Merck aluminum-backed $0.2 \mathrm{~mm}$ silica gel 60 F-254 plates. Merck silica gel 60 (230400mesh) samples were used in column chromatography experiments. Varian VNMRS-400 MHz and Perkin Elmer FT-IR spectrometers were employed to record infrared (IR) and ${ }^{1}$ HNMR spectra of the products obtained in this study. Chemical shifts are reported as values in ppm relative to $\mathrm{CHCl}_{3}(7.26)$, and TMS was used as internal standard.

\section{General procedure for synthesis of nitroarenes}

Ten (10) mmol of the substrate (aromatic compounds), one (1.0) $\mathrm{mmol}_{\text {of }} \mathrm{KHSO}_{4}$, one (1.0) mmol of Peroxide in an acetonitrile solvent and $2.0 \mathrm{mmol} \mathrm{NaNO}_{2}$ were taken in a round bottom flask and the mixture was stirred under reflux conditions. The progress of the reaction was monitored by TLC. After completion of the reaction, the reaction mixture was treated with a $\mathrm{NaHCO}_{3}$ solution. The organic layer was separated, dried over $\mathrm{Na}_{2} \mathrm{SO}_{4}$, and evaporated under vacuum. The crude product was purified by column chromatography using ethyl acetate -hexane as eluant to get pure product. Products thus obtained are characterized as nitro derivatives by spectroscopic methods.

\section{Microwave assisted synthesis of nitroarenes}

The constituents of the reaction such as $10 \mathrm{mmol}$ of the substrate, $1 \mathrm{mmol}$ of $\mathrm{KHSO}_{4}, 1 \mathrm{mmol}$ of peroxide in acetonitrile solvent and $2.0 \mathrm{mmol} \mathrm{NaNO}_{2}$ were taken in a clean conical flask at room temperature and placed in a microwave oven. The progress of the reaction was monitored by TLC. After completion, the reaction mixture was further processed for the isolation of product as detailed previously.

\section{Grindstone synthesis of nitroarenes}

The constituents of the reaction such as $10 \mathrm{mmol}$ of the substrate, $1.0 \mathrm{mmol}$ of $\mathrm{KHSO}_{4}, 2.0 \mathrm{mmol}$ of $\mathrm{NaNO}_{2}$, and a catalytic amount $(1 \mathrm{mmol})$ of peroxide are taken in a mortar and ground with a pestle under solvent free conditions. The progress of the reaction was monitored by TLC. After completion, the workup procedure to obtain the products is largely similar to the one discussed in the earlier section. Obtained results from all the methods are presented Tables- 2 to 4. 
RASĀYAN $J$. Chem.

Vol. 10 | No. 3 |790 - 798 | July - September | 2017

Table-1: List of aromatic compounds and nitroarenes

\begin{tabular}{|c|c|c|c|}
\hline Substrate & Product & Substrate & Product \\
\hline (1a)Phenol & (2a) 2-NO ${ }_{2}$ Phenol & (1k)p-OH Phenol & (2k) 2- $\mathrm{NO}_{2}$ Benzene-1,4-diol \\
\hline (1b)o-Cresol & (2b) 2-Me- 4-NO $\mathrm{NO}_{2}$ Phenol & (1l)p-Nitro Phenol & (21) 2,4-di Nitro Phenol \\
\hline (1c)p-Cresol & (2c) 2-NO $\mathrm{NO}_{2} 4-\mathrm{Me}$ Phenol & (1m)p-Amino Phenol & (2m) 2-Nitro4-Amino Phenol \\
\hline (1d)m-Cresol & (2d) 3-Me- 4- $\mathrm{NO}_{2}$ Phenol & (1n) $\alpha$-Naphthol & (2n) 2- $\mathrm{NO}_{2}-1-\mathrm{Naphthol}$ \\
\hline (1e)o-Cl Phenol & (2e) 4-NO $\mathrm{NO}_{2} 2-\mathrm{Cl}$ Phenol & (1o) $\beta$-Naphthol & (2o) 1-NO 2 -2-Naphthol \\
\hline (1f)m-Cl Phenol & (2f) 4-NO $\mathrm{NO}_{2} 3-\mathrm{Cl}$ Phenol & (1p)Aniline & (2p) 4-Nitro Aniline \\
\hline$(1 g) p$-Cl Phenol & (2g) 2-NO ${ }_{2} 4-\mathrm{Cl}$ Phenol & (1q)Salicylic acid & (2q) 2-OH 4-NO $\mathrm{NO}_{2}$ Benzoic acid \\
\hline (1h)p-Br Phenol & (2h) 2-NO $\mathrm{NO}_{2} 4-\mathrm{Br}$ Phenol & (1r)Furan & (2r) 2-NO ${ }_{2}$ furan \\
\hline (1i) $O$-OH Phenol & (2i) 4- $\mathrm{NO}_{2}$ Benzene-1,2-diol & (1s)Thiophene & (2s) $2-\mathrm{NO}_{2}$ thiophene \\
\hline (1j)m-OH Phenol & (2j) 4- $\mathrm{NO}_{2}$ Benzene-1,3-diol & (1t)Pyrrole & (2t) $2-\mathrm{NO}_{2}$ Pyrrole \\
\hline
\end{tabular}

Table-2: Peroxy compound/NaNO 2 driven Nitration of Aromatic compounds in aq. $\mathrm{KHSO}_{4}$ medium

\begin{tabular}{l|l|l|l|l|l|l|l|l|l|l|l}
\hline \multicolumn{2}{l}{ Entry codes } & \multicolumn{2}{l}{ PDS } & \multicolumn{2}{l}{ PMS } & \multicolumn{2}{l}{$\mathrm{H}_{2} \mathrm{O}_{2}$} & \multicolumn{2}{l}{ TBHP } & \multicolumn{2}{l}{ SPB } \\
\hline Substrate & Product & $\begin{array}{l}\text { RT } \\
(\mathrm{hr})\end{array}$ & $\begin{array}{l}\% \\
\text { Yield }\end{array}$ & $\begin{array}{l}\text { RT } \\
(\mathrm{hr})\end{array}$ & $\begin{array}{l}\% \\
\text { Yield }\end{array}$ & $\begin{array}{l}\text { RT } \\
(\mathrm{hr})\end{array}$ & $\begin{array}{l}\% \\
\text { Yield }\end{array}$ & $\begin{array}{l}\text { RT } \\
(\mathrm{hr})\end{array}$ & $\begin{array}{l}\% \\
\text { Yield }\end{array}$ & $\begin{array}{l}\text { RT } \\
(\mathrm{hr})\end{array}$ & $\begin{array}{l}\% \\
\text { Yield }\end{array}$ \\
\hline$(1 \mathrm{a})$ & $(2 \mathrm{a})$ & 2 & 70 & 4 & 70 & 5 & 70 & 5 & 68 & 7 & 68 \\
\hline$(1 \mathrm{~b})$ & $(2)$ & 2 & 70 & 4 & 68 & 5 & 70 & 5 & 70 & 7 & 70 \\
\hline$(1 \mathrm{c})$ & $(2 \mathrm{c})$ & 2 & 75 & 4 & 74 & 5 & 75 & 5 & 75 & 7 & 75 \\
\hline$(1 \mathrm{~d})$ & $(2 \mathrm{~d})$ & 2 & 70 & 4 & 68 & 5 & 65 & 5 & 65 & 7 & 65 \\
\hline$(1 \mathrm{e})$ & $(2 \mathrm{e})$ & 4 & 80 & 6 & 80 & 6 & 75 & 6 & 75 & 8 & 75 \\
\hline$(1 \mathrm{f})$ & $(2 \mathrm{f})$ & 4 & 78 & 6 & 78 & 6 & 75 & 6 & 75 & 8 & 75 \\
\hline$(1 \mathrm{~g})$ & $(2 \mathrm{~g})$ & 4 & 80 & 6 & 80 & 6 & 75 & 6 & 75 & 8 & 70 \\
\hline$(1 \mathrm{~h})$ & $(2 \mathrm{~h})$ & 4 & 75 & 6 & 77 & 6 & 75 & 6 & 75 & 8 & 74 \\
\hline$(1 \mathrm{i})$ & $(2 \mathrm{i})$ & 3 & 85 & 4 & 80 & 5 & 80 & 5 & 78 & 7 & 75 \\
\hline$(1 \mathrm{j})$ & $(2 \mathrm{j})$ & 3 & 80 & 4 & 77 & 5 & 80 & 5 & 80 & 7 & 75 \\
\hline$(1 \mathrm{k})$ & $(2 \mathrm{k})$ & 3 & 80 & 4 & 80 & 5 & 78 & 5 & 78 & 7 & 75 \\
\hline$(11)$ & $(2 \mathrm{l})$ & 4 & 75 & 6 & 75 & 7 & 75 & 8 & 70 & 10 & 70 \\
\hline$(1 \mathrm{~m})$ & $(2 \mathrm{~m})$ & 3 & 80 & 6 & 80 & 7 & 78 & 7 & 75 & 9 & 75 \\
\hline$(1 \mathrm{n})$ & $(2 \mathrm{n})$ & 2 & 85 & 4 & 80 & 4 & 80 & 4 & 80 & 5 & 80 \\
\hline$(1 \mathrm{o})$ & $(2 \mathrm{o})$ & 3 & 85 & 5 & 80 & 5 & 80 & 5 & 75 & 6 & 75 \\
\hline$(1 \mathrm{p})$ & $(2 \mathrm{p})$ & 2 & 75 & 4 & 75 & 5 & 75 & 5 & 70 & 5 & 70 \\
\hline$(1 \mathrm{q})$ & $(2 \mathrm{q})$ & 3 & 75 & 5 & 75 & 6 & 75 & 7 & 75 & 7 & 75 \\
\hline$(1 \mathrm{r})$ & $(2 \mathrm{r})$ & 2 & 70 & 4 & 70 & 5 & 70 & 5 & 68 & 6 & 65 \\
\hline$(1 \mathrm{~s})$ & $(2 \mathrm{~s})$ & 2 & 75 & 4 & 75 & 5 & 73 & 5 & 70 & 6 & 70 \\
\hline$(1 \mathrm{t})$ & $(2 \mathrm{t})$ & 1 & 80 & 3 & 80 & 4 & 75 & 4 & 70 & 4 & 70 \\
\hline
\end{tabular}

Table-3: Grindstone protocol for Peroxy compound/NaNO $2 / \mathrm{KHSO}_{4}$ driven Nitration of Aromatic compounds under solvent-free conditions

\begin{tabular}{|c|c|c|c|c|c|c|c|c|c|c|c|}
\hline \multicolumn{2}{|c|}{ Entry codes } & \multicolumn{2}{|l|}{ PDS } & \multicolumn{2}{|l|}{ PMS } & \multicolumn{2}{|l|}{$\mathrm{H}_{2} \mathrm{O}_{2}$} & \multicolumn{2}{|c|}{ TBHP } & \multicolumn{2}{|l|}{ SPB } \\
\hline Substrate & Product & $\begin{array}{l}\text { RT } \\
(\mathrm{min})\end{array}$ & $\begin{array}{l}\% \\
\text { Yield }\end{array}$ & $\begin{array}{l}\text { RT } \\
(\mathrm{min})\end{array}$ & $\begin{array}{l}\% \\
\text { Yield }\end{array}$ & $\begin{array}{l}\text { RT } \\
(\mathrm{min})\end{array}$ & $\begin{array}{l}\% \\
\text { Yield }\end{array}$ & $\begin{array}{l}\text { RT } \\
\text { (min) }\end{array}$ & $\begin{array}{l}\% \\
\text { Yield }\end{array}$ & $\begin{array}{l}\text { RT } \\
(\mathrm{min})\end{array}$ & $\begin{array}{l}\% \\
\text { Yield }\end{array}$ \\
\hline (1a) & (2a) & 50 & 70 & 120 & 70 & 150 & 70 & 160 & 65 & 180 & 70 \\
\hline (1b) & (2) & 50 & 70 & 90 & 70 & 120 & 70 & 120 & 70 & 160 & 68 \\
\hline (1c) & (2c) & 50 & 75 & 90 & 75 & 120 & 75 & 120 & 70 & 160 & 75 \\
\hline
\end{tabular}


RASĀYAN J. Chem.

Vol. 10 | No. 3 |790 - 798 | July - September | 2017

\begin{tabular}{l|l|l|l|l|l|l|l|l|l|l|l}
\hline$(1 \mathrm{~d})$ & $(2 \mathrm{~d})$ & 50 & 70 & 100 & 70 & 130 & 68 & 130 & 65 & 180 & 68 \\
\hline$(1 \mathrm{e})$ & $(2 \mathrm{e})$ & 100 & 80 & 150 & 80 & 180 & 77 & 190 & 75 & 220 & 75 \\
\hline$(1 \mathrm{f})$ & $(2 \mathrm{f})$ & 100 & 80 & 180 & 75 & 190 & 75 & 200 & 70 & 240 & 75 \\
\hline$(1 \mathrm{~g})$ & $(2 \mathrm{~g})$ & 100 & 80 & 150 & 78 & 180 & 75 & 190 & 75 & 220 & 77 \\
\hline$(1 \mathrm{~h})$ & $(2 \mathrm{~h})$ & 110 & 75 & 180 & 78 & 200 & 75 & 210 & 70 & 240 & 75 \\
\hline$(1 \mathrm{i})$ & $(2 \mathrm{i})$ & 70 & 85 & 90 & 83 & 120 & 80 & 120 & 78 & 160 & 80 \\
\hline$(1 \mathrm{j})$ & $(2 \mathrm{j})$ & 70 & 80 & 90 & 80 & 120 & 80 & 120 & 75 & 180 & 75 \\
\hline$(1 \mathrm{k})$ & $(2 \mathrm{k})$ & 70 & 80 & 90 & 80 & 120 & 79 & 120 & 79 & 160 & 80 \\
\hline$(1 \mathrm{l})$ & $(2 \mathrm{l})$ & 100 & 80 & 150 & 78 & 200 & 77 & 210 & 75 & 270 & 75 \\
\hline$(1 \mathrm{~m})$ & $(2 \mathrm{~m})$ & 70 & 80 & 120 & 80 & 180 & 79 & 180 & 75 & 240 & 75 \\
\hline$(1 \mathrm{n})$ & $(2 \mathrm{n})$ & 50 & 85 & 90 & 80 & 100 & 80 & 100 & 78 & 120 & 80 \\
\hline$(1 \mathrm{o})$ & $(2 \mathrm{o})$ & 70 & 85 & 90 & 80 & 120 & 80 & 120 & 75 & 150 & 75 \\
\hline$(1 \mathrm{p})$ & $(2 \mathrm{p})$ & 50 & 75 & 75 & 75 & 120 & 75 & 120 & 70 & 120 & 70 \\
\hline$(1 \mathrm{q})$ & $(2 \mathrm{q})$ & 80 & 75 & 150 & 75 & 200 & 75 & 200 & 70 & 240 & 75 \\
\hline$(1 \mathrm{r})$ & $(2 \mathrm{r})$ & 50 & 70 & 80 & 72 & 120 & 70 & 120 & 68 & 150 & 68 \\
\hline$(1 \mathrm{~s})$ & $(2 \mathrm{~s})$ & 50 & 75 & 90 & 74 & 130 & 73 & 130 & 70 & 180 & 70 \\
\hline$(1 \mathrm{t})$ & $(2 \mathrm{t})$ & 30 & 80 & 60 & 80 & 90 & 76 & 90 & 75 & 120 & 75 \\
\hline
\end{tabular}

\section{RESULTS AND DISCUSSION}

Nitration of aromatic compounds using $\mathrm{NaNO}_{2}$ in the presence of Peroxide reagent $/ \mathrm{KHSO}_{4}$ under acid-free conventional conditions

Potassium hydrogen sulfate $\left(\mathrm{KHSO}_{4}\right)$ is a reusable, green and efficient solid catalyst for various organic reactions. Heravi et al earlier accomplished that certain phenols and naphthols can be conveniently nitrated regioselectively with $\mathrm{NaNO}_{2}$ using $\mathrm{KHSO}_{4}$ as a catalyst in good yields. ${ }^{38}$ The reaction times for most of the studied reactions are in the range of 5 to $7 \mathrm{hrs}$, depending on the structure of the substrate. In an effort to further enhance the reaction rates (i.e., further reduce the reaction times) we have used peroxy compounds $\left(\mathrm{H}_{2} \mathrm{O}_{2}\right.$, TBHP, PDS, PMS or SPB) as additives under conventional and non-conventional conditions under mineral acid-free medium. Spectroscopic data of isolated products are in agreement with our earlier reports.

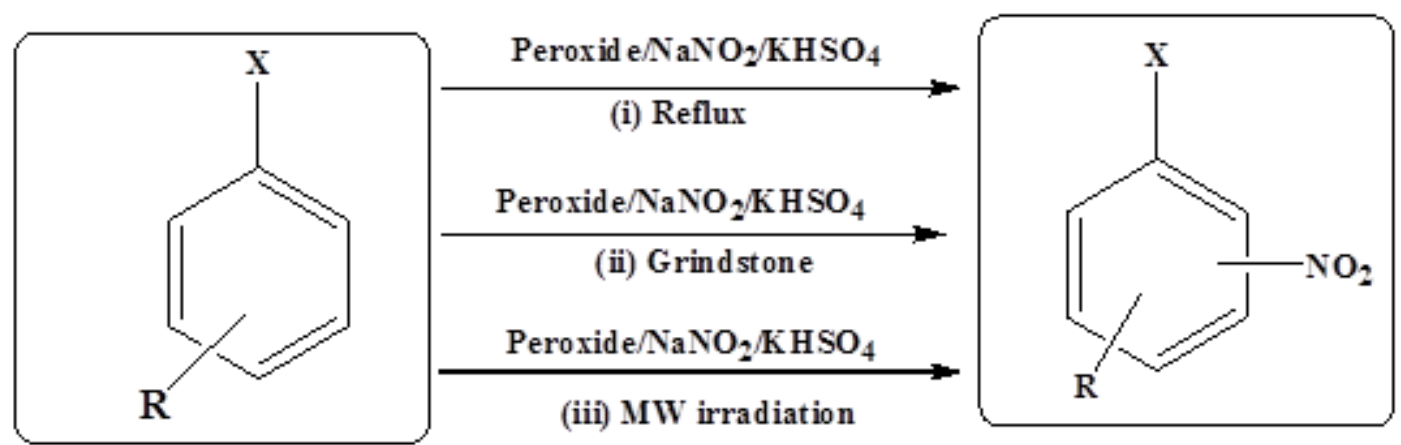

Where $\mathrm{X}=-\mathrm{OH}$ or $-\mathrm{NH}_{2}$; Peroxide Reagent $=\mathrm{PDS}, \mathrm{PMS}, \mathrm{H}_{2} \mathrm{O}_{2}, \mathrm{SPB}, \mathrm{TBHP}$

Scheme-1: Mono Nitration of Aromatic Compound using NaNO2 in presence of KHSO4 / Peroxide Reagent

It is well known that the peroxy compounds $\left(\mathrm{H}_{2} \mathrm{O}_{2}\right.$, TBHP, PDS, PMS or SPB) form free-radical intermediates in situ during the course of several reactions. In order to detect the formation of free-radical intermediates pertaining to these peroxide reagents, olefinic monomers such as freshly prepared acrylamide or deaerated acrylonitrile were added to the reaction mixture under a nitrogen atmosphere. But the peroxy compounds $\left(\mathrm{H}_{2} \mathrm{O}_{2}\right.$, TBHP, PDS, PMS or SPB) used in this study did not initiate/induce 
polymerization of added olefinic monomers even after $24 \mathrm{hrs}$ under reflux conditions. This observation may indicate the absence of free radical intermediates. In the present study, $\mathrm{KHSO}_{4}$ creates an acidic environment due to the protolysis of the bisulfate ion $\left(\mathrm{HSO}_{4}^{-}\right)$followed by the release of a proton $\left(\mathrm{H}^{+}\right)$ without decomposition and thus acts as a pool of proton source. ${ }^{36-39}$ Basically, all the peroxy compounds in this study are strong oxidizing agents, which may present as free radical or ionic species. The absence of free radical species, thus suggests the participation of either the ionic species or molecular species in the mechanism of generating active nitronium ion ingredient as shown in the following steps:

\section{Step-I: Generation of Nitronium ion electrophile}

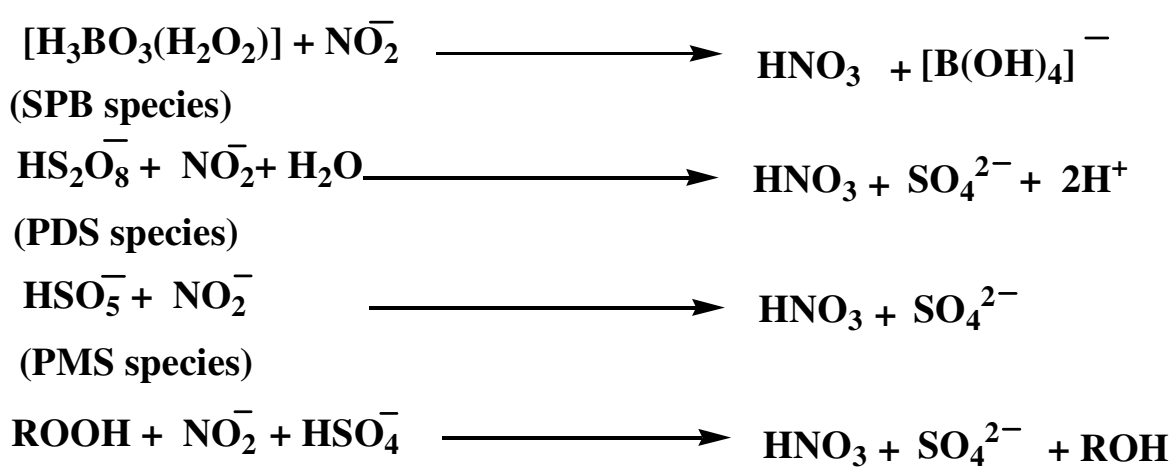

(Peroxide species; $\mathbf{R}=\mathbf{H}$ for $\mathbf{H P}$, tert- butyl for TBHP )

Active nitronium ion $\left(\mathrm{NO}_{2}{ }^{+}\right)$electrophile is then generated due to the reaction between $\mathrm{HNO}_{3}$ and $\mathrm{KHSO}_{4}$ according to the following reaction,

$$
\mathrm{HNO}_{3}+\mathrm{HSO}_{4}^{-} \longrightarrow \mathrm{NO}_{2}{ }^{+}+\mathrm{H}_{2} \mathrm{O}+\mathrm{SO}_{4}{ }^{2-}
$$

Step-II: Nitronium ion $\left(\mathrm{NO}_{2}{ }^{+}\right)$thus generated attacks the aromatic ring to afford nitroarene according to the following scheme-1,

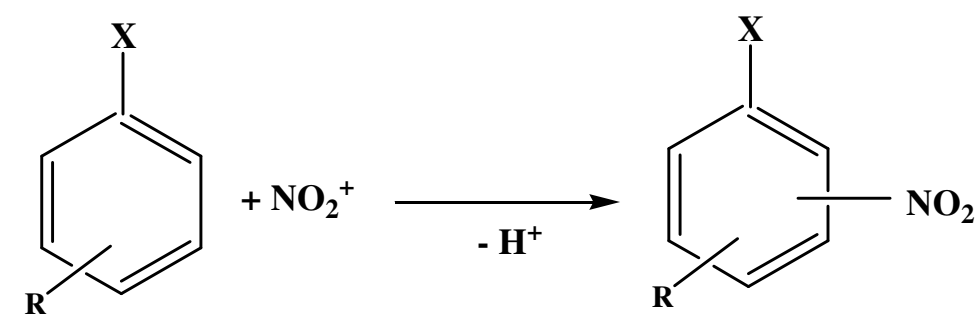

(Where R = EDG or EWG; $\mathrm{X}=$ functional group)

Scheme-2

Table-4: Microwave assisted Nitration of Aromatic compounds using Peroxy compound/ $\mathrm{NaNO}_{2} / \mathrm{KHSO}_{4}$ under solvent-free conditions

\begin{tabular}{l|l|l|l|l|l|l|l|l|l|l|l}
\hline \multicolumn{2}{l}{ Entry codes } & \multicolumn{2}{l|}{ PDS } & \multicolumn{2}{l|}{ PMS } & \multicolumn{2}{l}{$\mathrm{H}_{2} \mathrm{O}_{2}$} & \multicolumn{2}{l}{ TBHP } & \multicolumn{2}{l}{ SPB } \\
\hline Substrate & Product & $\begin{array}{l}\text { RT } \\
(\mathrm{sec})\end{array}$ & $\begin{array}{l}\% \\
\text { Yield }\end{array}$ & $\begin{array}{l}\text { RT } \\
(\mathrm{sec})\end{array}$ & $\begin{array}{l}\% \\
\text { Yield }\end{array}$ & $\begin{array}{l}\text { RT } \\
(\mathrm{sec})\end{array}$ & $\begin{array}{l}\% \\
\text { Yield }\end{array}$ & $\begin{array}{l}\text { RT } \\
(\mathrm{sec})\end{array}$ & $\begin{array}{l}\% \\
\text { Yield }\end{array}$ & $\begin{array}{l}\text { RT } \\
(\mathrm{sec})\end{array}$ & $\begin{array}{l}\% \\
\text { Yield }\end{array}$ \\
\hline$(1 \mathrm{a})$ & $(2 \mathrm{a})$ & 60 & 75 & 60 & 72 & 70 & 70 & 70 & 65 & 90 & 70 \\
\hline$(1 \mathrm{~b})$ & $(2)$ & 40 & 75 & 40 & 70 & 50 & 70 & 60 & 70 & 70 & 70 \\
\hline$(1 \mathrm{c})$ & $(2 \mathrm{c})$ & 40 & 80 & 40 & 78 & 50 & 75 & 60 & 72 & 70 & 75 \\
\hline$(1 \mathrm{~d})$ & $(2 \mathrm{~d})$ & 40 & 75 & 40 & 73 & 50 & 70 & 60 & 70 & 75 & 70 \\
\hline$(1 \mathrm{e})$ & $(2 \mathrm{e})$ & 50 & 82 & 70 & 80 & 80 & 79 & 85 & 75 & 90 & 75 \\
\hline
\end{tabular}


RASĀYAN J. Chem.

Vol. 10 | No. 3 |790 - 798 | July - September | 2017

\begin{tabular}{l|l|l|l|l|l|l|l|l|l|l|l}
\hline$(1 \mathrm{f})$ & $(2 \mathrm{f})$ & 60 & 80 & 80 & 77 & 90 & 75 & 95 & 72 & 100 & 75 \\
\hline$(1 \mathrm{~g})$ & $(2 \mathrm{~g})$ & 50 & 80 & 70 & 80 & 80 & 78 & 85 & 75 & 90 & 78 \\
\hline$(1 \mathrm{~h})$ & $(2 \mathrm{~h})$ & 60 & 75 & 80 & 78 & 100 & 75 & 110 & 72 & 120 & 75 \\
\hline$(1 \mathrm{i})$ & $(2 \mathrm{i})$ & 40 & 87 & 50 & 85 & 60 & 82 & 65 & 80 & 65 & 82 \\
\hline$(1 \mathrm{j})$ & $(2 \mathrm{j})$ & 45 & 82 & 60 & 80 & 65 & 80 & 70 & 75 & 75 & 75 \\
\hline$(1 \mathrm{k})$ & $(2 \mathrm{k})$ & 40 & 85 & 50 & 83 & 60 & 80 & 65 & 80 & 65 & 82 \\
\hline$(1 \mathrm{l})$ & $(2 \mathrm{l})$ & 100 & 82 & 140 & 80 & 160 & 78 & 180 & 75 & 180 & 78 \\
\hline$(1 \mathrm{~m})$ & $(2 \mathrm{~m})$ & 50 & 85 & 55 & 82 & 60 & 80 & 70 & 78 & 90 & 80 \\
\hline$(1 \mathrm{n})$ & $(2 \mathrm{n})$ & 30 & 88 & 40 & 85 & 45 & 82 & 50 & 80 & 60 & 80 \\
\hline$(1 \mathrm{o})$ & $(2 \mathrm{o})$ & 50 & 88 & 60 & 85 & 65 & 80 & 65 & 80 & 70 & 80 \\
\hline$(1 \mathrm{p})$ & $(2 \mathrm{p})$ & 30 & 80 & 40 & 80 & 45 & 78 & 50 & 75 & 60 & 75 \\
\hline$(1 \mathrm{q})$ & $(2 \mathrm{q})$ & 60 & 80 & 70 & 75 & 80 & 75 & 90 & 75 & 120 & 75 \\
\hline$(1 \mathrm{r})$ & $(2 \mathrm{r})$ & 40 & 75 & 50 & 74 & 60 & 73 & 60 & 70 & 60 & 70 \\
\hline$(1 \mathrm{~s})$ & $(2 \mathrm{~s})$ & 40 & 75 & 45 & 77 & 50 & 75 & 50 & 70 & 60 & 72 \\
\hline$(1 \mathrm{t})$ & $(2 \mathrm{t})$ & 30 & 85 & 40 & 85 & 45 & 80 & 45 & 80 & 55 & 82 \\
\hline
\end{tabular}

The data presented in Tables-2 to 4 revealed that the reaction times (R.T) in microwave assisted (MWA) reactions reduced to a few seconds ( 30 to $50 \mathrm{sec}$ ) from 2 to $7 \mathrm{hrs}$ (under conventional methods), and 50 to 240 minutes with grindstone techniques at room temperature. Rate enhancements in MW assisted methods could be attributed to bulk activation of molecules because microwave irradiation directly supplements energy to all the reactant molecules present in the reaction vessel without heating the reactor as explained in earlier literature reports..$^{50-53}$ On the other hand, rate accelerations under grindstone conditions could be explained due to bulk activation of the molecule due to the heat energy generated due to frictional forces operated during the course of grinding by pestle in a mortar ${ }^{46-49}$. Observed data further indicate that nitration followed o/p selectivity principle.

\section{Spectral data of certain compounds:}

1. 2-NO 2 Phenol: ${ }^{1} \mathrm{HNMR}\left(300 \mathrm{MHz}, \mathrm{CDCl}_{3}\right): \delta 9.56(\mathrm{~s}, 1 \mathrm{H}, \mathrm{OH}), 8.19(\mathrm{dd}, 1 \mathrm{H}, \mathrm{J}=9.1 \mathrm{~Hz}, \mathrm{~J}=7 \mathrm{~Hz}), 7.59$ $(\mathrm{s}, 1 \mathrm{H}, \mathrm{J}=8 \mathrm{~Hz} \mathrm{~J}=7.5 \mathrm{~Hz}) 6.99(\mathrm{~d}, 1 \mathrm{H}, \mathrm{J}=8.5 \mathrm{~Hz}) 9.18(\mathrm{~d}, 1 \mathrm{H}, \mathrm{J}=8 \mathrm{~Hz})$; $\mathrm{m} / \mathrm{z}=139 ;$ Melting Point $=45-49^{\circ} \mathrm{C}$

2. 2-Me- 4-NO ${ }_{2}$ Phenol: ${ }^{1} \mathrm{HNMR}\left(300 \mathrm{MHz}, \mathrm{CDCl}_{3}\right): \delta 10.55(\mathrm{~s}, 1 \mathrm{H}, \mathrm{OH}), 2.35(\mathrm{~s}, 3 \mathrm{H}, \mathrm{Me}), 6.85(\mathrm{~d}, 1 \mathrm{H}$, $\mathrm{J}=8.5 \mathrm{~Hz}) 8.12(\mathrm{~d}, 1 \mathrm{H}, \mathrm{J}=8.5 \mathrm{~Hz}) 8.25(\mathrm{dd}, 1 \mathrm{H}, \mathrm{J}=8.5 \mathrm{~Hz}, \mathrm{~J}=7.5 \mathrm{~Hz})$;

$\mathrm{m} / \mathrm{z}=153$; Melting Point $=95-98^{\circ} \mathrm{C}$

3. 2-NO 4 4-Me Phenol: ${ }^{1} \mathrm{HNMR}\left(300 \mathrm{MHz}, \mathrm{CDCl}_{3}\right)$ : $\delta 10.42$ (s, $\left.1 \mathrm{H}, \mathrm{OH}\right), 2.42(\mathrm{~s}, 3 \mathrm{H}, \mathrm{Me}), 7.32(\mathrm{~d}, 1 \mathrm{H}$, $\mathrm{J}=8 \mathrm{~Hz}), 7.12(\mathrm{~d}, 1 \mathrm{H}, \mathrm{J}=8 \mathrm{~Hz}), 7.92(\mathrm{~s}, 1 \mathrm{H}) ; \mathrm{m} / \mathrm{z}=153 ;$ Melting Point $=32-34^{\circ} \mathrm{C}$

4. 3-Me- 4-NO Phenol: $^{1} \mathrm{HNMR}\left(300 \mathrm{MHz}, \mathrm{CDCl}_{3}\right)$ : $\delta 10.64(\mathrm{~s}, 1 \mathrm{H}, \mathrm{OH}), 2.35(\mathrm{~s}, 3 \mathrm{H}, \mathrm{Me}), 6.85(\mathrm{~d}, 1 \mathrm{H}$, $\mathrm{J}=8 \mathrm{~Hz}), 8.12(\mathrm{~d}, 1 \mathrm{H}, \mathrm{J}=8 \mathrm{~Hz}), 6.76(\mathrm{~s}, 1 \mathrm{H}) ; \mathrm{m} / \mathrm{z}=153 ;$ Melting Point $=127-129^{\circ} \mathrm{C}$

5. 4-NO $\mathrm{O}_{2}$ 2-Cl Phenol: ${ }^{1} \mathrm{HNMR}\left(300 \mathrm{MHz}, \mathrm{CDCl}_{3}\right): \delta 10.66(\mathrm{~s}, 1 \mathrm{H}, \mathrm{OH}), 7.15(\mathrm{~d}, 1 \mathrm{H}, \mathrm{J}=8.5 \mathrm{~Hz}), 8.12(\mathrm{~d}$, $1 \mathrm{H}, \mathrm{J}=8.5 \mathrm{~Hz}), 8.34(\mathrm{~s}, 1 \mathrm{H}) ; \mathrm{m} / \mathrm{z}=174 ;$ Melting Point $=106-108^{\circ} \mathrm{C}$

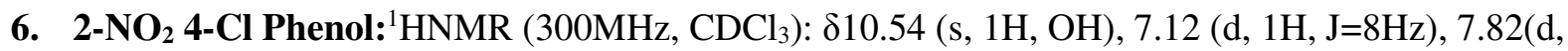
$1 \mathrm{H}, \mathrm{J}=8 \mathrm{~Hz}), 8.36(\mathrm{~s}, 1 \mathrm{H}) ; \mathrm{m} / \mathrm{z}=174 ;$ Melting Point $=88-90^{\circ} \mathrm{C}$ 
7. 2-NO $\mathrm{N}_{2}$ 4-Br Phenol: ${ }^{1} \mathrm{HNMR}\left(300 \mathrm{MHz}, \mathrm{CDCl}_{3}\right): \delta 10.45(\mathrm{~s}, 1 \mathrm{H}, \mathrm{OH}), 7.10(\mathrm{~d}, 1 \mathrm{H}, \mathrm{J}=8 \mathrm{~Hz}), 7.72(\mathrm{~d}$, $1 \mathrm{H}, \mathrm{J}=8 \mathrm{~Hz}), 8.25(\mathrm{~s}, 1 \mathrm{H}) ; \mathrm{m} / \mathrm{z}=218 ;$ Melting Point $=92-96^{\circ} \mathrm{C}$

8. 4-NH $\mathrm{NH}_{2}$-2- $\mathrm{NO}_{2}$ Phenol: ${ }^{1} \mathrm{H}$ NMR $\left(300 \mathrm{MHz}, \mathrm{CDCl}_{3}\right)$ : $\delta 4.45(\mathrm{~s}, 2 \mathrm{H}) 9.92(\mathrm{~s}, 1 \mathrm{H}) 6.76(\mathrm{~d}, \mathrm{~J}=8 \mathrm{~Hz}, 1 \mathrm{H})$, $6.82(\mathrm{~d}, \mathrm{~J}=8 \mathrm{~Hz}, 1 \mathrm{H}), 7.24(\mathrm{~s}, 1 \mathrm{H}) ; \mathrm{m} / \mathrm{z}=154 ;$ Melting Point $=125-128^{\circ}$

9. 4- $\mathrm{NO}_{2}$ aniline: ${ }^{1} \mathrm{H} \mathrm{NMR}\left(300 \mathrm{MHz}, \mathrm{CDCl}_{3}\right): \delta 7.94(\mathrm{~d}, \mathrm{~J}=5.5 \mathrm{~Hz}, 2 \mathrm{H}), 6.76(\mathrm{~d}, \mathrm{~J}=5.49 \mathrm{~Hz}, 2 \mathrm{H})$, 4.25 (brs, $2 \mathrm{H}$ ); $\mathrm{m} / \mathrm{z}=138$; Melting Point $=148-150^{\circ} \mathrm{C}$

10. 2- $\mathrm{NO}_{2}$-1-Naphthol: ${ }^{1} \mathrm{HNMR}\left(300 \mathrm{MHz}, \mathrm{CDCl}_{3}\right)$ : $\delta 12.24(\mathrm{~s}, 1 \mathrm{H}, \mathrm{OH}), 7.66(\mathrm{~m}, 1 \mathrm{H}), 7.76(\mathrm{~m}, 1 \mathrm{H})$, $8.58(\mathrm{~d}, 1 \mathrm{H}, \mathrm{J}=8.5 \mathrm{~Hz}), 8.05(\mathrm{~d}, 1 \mathrm{H}, \mathrm{J}=8.5 \mathrm{~Hz}, \mathrm{~J}=4 \mathrm{~Hz}), 8.15(\mathrm{~d}, 1 \mathrm{H}, \mathrm{J}=8.5 \mathrm{~Hz}), 7.44(\mathrm{~d}, 1 \mathrm{H}, \mathrm{J}=9 \mathrm{~Hz}) ; \mathrm{m} / \mathrm{z}=$ 189 ; Melting Point $=124-126^{\circ} \mathrm{C}$

11. 1-NO 2 -2-Naphthol: ${ }^{1} \mathrm{HNMR}(300 \mathrm{MHz}, \mathrm{CDCl} 3)$ : $\delta 12.18(\mathrm{~s}, 1 \mathrm{H}, \mathrm{OH}), 7.58(\mathrm{~m}, 1 \mathrm{H}), 7.80(\mathrm{~m}, 1 \mathrm{H})$, $7.20(\mathrm{~d}, 1 \mathrm{H}, \mathrm{J}=9 \mathrm{~Hz}), 8.10(\mathrm{~m}, 1 \mathrm{H}), 7.68(\mathrm{dd}, 1 \mathrm{H}, \mathrm{J}=8.25 \mathrm{~Hz} \mathrm{~J}=5 \mathrm{~Hz}), 8.65(\mathrm{~d}, 1 \mathrm{H}, \mathrm{J}=9 \mathrm{~Hz})$; $\mathrm{m} / \mathrm{z}=189 ;$ Melting Point $=98-100^{\circ} \mathrm{C}$

12. 2- $\mathrm{NO}_{2}$ furan: ${ }^{1} \mathrm{H} \mathrm{NMR}\left(300 \mathrm{MHz}, \mathrm{CDCl}_{3}\right): \delta 6.68(\mathrm{dd}, \mathrm{J}=3.5 \mathrm{~Hz}, \mathrm{~J} 1.75 \mathrm{~Hz}, 1 \mathrm{H}), 7.34(\mathrm{dd}, \mathrm{J}=3.5 \mathrm{~Hz}, \mathrm{~J}=$ $1.0 \mathrm{~Hz}, 1 \mathrm{H}), 7.57(\mathrm{dd}, \mathrm{J}=1.75 \mathrm{~Hz}, \mathrm{~J}=1.0 \mathrm{~Hz}, 1 \mathrm{H}) ; \mathrm{m} / \mathrm{z}=113$;

Boiling Point $=85-88^{\circ} \mathrm{C}$

13. 2- $\mathrm{NO}_{2}$ thiophene: ${ }^{1} \mathrm{H}$ NMR $\left(300 \mathrm{MHz}, \mathrm{CDCl}_{3}\right): \delta 7.07(\mathrm{q}, \mathrm{J}=4.25 \mathrm{~Hz}, \mathrm{~J}=5.25 \mathrm{~Hz}, 1 \mathrm{H}), 7.55(\mathrm{dd}, \mathrm{J}=$ $1.75 \mathrm{~Hz}, \mathrm{~J}=5.25 \mathrm{~Hz}, 1 \mathrm{H}), 7.93(\mathrm{dd}, \mathrm{J}=1.75 \mathrm{~Hz}, \mathrm{~J}=4.25 \mathrm{~Hz}, 1 \mathrm{H}) ; \mathrm{m} / \mathrm{z}=129$;

Melting Point $=44-47^{\circ} \mathrm{C}$

\section{CONCLUSION}

The authors have accomplished peoxycompounds such as $\mathrm{H}_{2} \mathrm{O}_{2}$, peroxydisulfate (PDS), peroxymonosulfate (PMS), sodium perborate (SPB), and tert-butyl hydroperoxide (TBHP) as efficient catalysts for nitration of aromatic and heteroaromatic compounds in presence of $\mathrm{NaNO}_{2} / \mathrm{KHSO}_{4}$ under mineral acid free conventional and non-conventional methods such as microwave irradiation and grindstone techniques. Reaction times reduced from (2 to 7 ) hours to (25 to 60) minutes from conventional to grindstone method, which in turn reduced to (25 to 120) seconds in microwave assisted reactions. Product yields are also increased from 6 to $10 \%$ under microwave irradiation as compared to conventional procedures. All the synthesized compounds were characterized by ${ }^{1} \mathrm{H}$ NMR and mass spectral studies.

\section{REFERENCES}

1. (a) G.A. Olah, R. Malhotra, S.C. Narang, "Nitration Methods and Mechanisms," VCH, New York, (1989); (b) G.A. Olah, S.C. Narang, J.A. Olah, K. Lammertsma, Proc. Natl. Acad. Sci. USA., 79, 4487(1982).

2. J.G. Hoggett, R.B. Monodie, J.R. Penton, K. Scho-field, "Nitration and Aromatic Reactivity," Cambridge University Press, London, (1971).

3. (a) N. Ono, "The Nitro Group in Organic Synthesis," Wiley-VCH, New York, (2001); (b) J. March, M. B. Smith, "March's Advanced Organic Chemistry: Reactions, Mechanisms and Structure", John Wiley and Sons, Inc. 2007, Sixth Ed.

4. (a) J. M. Riego, Zeno Sedin, J. M. Zaldlvar, C. N. Marzianot, C.Tortatot, Tetrahedron Lett., 37(4), 513(1996); (b) F. J.Waller, A. G. M. Barrett, D. C. Braddock, D. Ramprasad, Chem. Commun. 613(1997); (c) J. W. Francis, Anthony, G. M. Barrett, D. Christopher Braddock, Dorai Ramprasad. Tetrahedron Lett., 39, 1641(1998).

5. A.P. Zaraisky, O.I. Kachurin, L.I. Velichko, I.A.Tikhonova, G.G. Furin, V.B. Shur, J. Mol. Catal. A: Chemical, 231,103(2005).

6. K. A. Christopher and Yan Zhang, Tetrahedron Lett., 53, 1592(2012).

7. T.E Sakkidurai and K. Pitchumani, J. Mol. Catal. A: Chemical, 185, 305(2002). 
8. Ahmed Kamal, B. Ashwini Kumar, Arifuddin, Midoux Patrick, Ultrason. Sonochem., 11, 455(2004).

9. S.Nitin, Nandurkar, M. J. Bhanushali, R. S. Jagtap, M. B. Bhalchandra, Ultrason. Sonochem., 14, 41(2007).

10. Gracy Elias. B. J. Mincher, S. P. Mezyk, JimMuller. L. R. Martin,Rad. Phys. Chem., 80, 554(2011).

11. P. D. Sharda, S. K. Vijay, K. D. Mohan, Catal. Comm., 3, 67(2002).

12. S.M. Kemdeoa, V.S. Sapkalb, G.N. Chaudharia, J. Mol. Catal. A: Chemical, 323, 70(2010).

13. V.V. Brei, S.V. Prudius, O.V. Melezhyk, Appl. Catal. A, 239, 11(2003).

14. A.S. Chhatre, R.A. Joshi, B.D. Kulkarni, J. Coll. Inter Sc., 158, 183(1993).

15. G. Yang, J. Shi, J. Li, Korean J. Chem. Engg., 20, 886(2003).

16. Masashi Hojo, Ryosuke Kato, Akio Narutaki, Tetsuya Maeda, Yuki Uji-yie.J. Mol. Liq., 163, 161(2011).

17. A. R. Hajipoura and E. R. Arnold, Tetrahedron Lett., 46, 8307(2005).

18. Wan-Po Yin and Min Shi, Tetrahedron, 61, 10861(2005).

19. A. K. Bose, S. N. Ganguly, V. Srirajan, A. H. Sharma, N. Lavlinskaia, M. S. Manhas. Symposia Division of Environmental Chemistry-American Chemical Society, 39, 453(1999).

20. A. K. Bose, S N. Ganguly, M. S. Manhas, H. William, Jeffrey Speck, Tetrahedron Lett., 47, 3213(2006).

21. M. M. Heravi, T. Benmorad, K. Bakhtiari, F. F. Bamoharram, H. A. Oskooie, J. Mol. Cal. A: Chem., 264, 318(2007).

22. (a) J. M. Melior and Raehel Parkes, Tetrahedron Lett., 38(50), 8739(1997); (b) Xianghua Yang, Chanjuan Xi , Yanfeng Jiang, Tetrahedron Lett., 46, 8781(2005); (c) H. M. Chawla and R. S. Mittal, Synthesis, 70 (1985); (d) S. Dincturk and J. H. Ridd, J. Chem. Soc., Perkin Trans., 2, 961(1982); (e) S. Dincturk and J. H. Ridd, J. Chem. Soc., Perkin Trans., 2, 965(1982); (f) A. Sridhar Rao, P. Srinivas, K. Suresh Babu, J. Madhusudana Rao, Tetrahedron Lett., 46, 8141(2005).

23. (a) J. Jonathan, G. K. S. Narayana Kumar, K. L. Kenneth, Tetrahedron Lett., 53, 6782(2012); ( b) R. R. Yadav, R. A. Vishwakarma, B. Sandip, Tetrahedron Lett., 53, 5958(2012); (c) S. Manna, S. Maity, S. Rana, S. Agasti, D. Maiti, Org. Lett., 14, 1736(2012); (d) Hong-Bin Sun, Ruimao Hua, Yingwu Yin, J. Org. Chem., 70, 9071(2005); (e) Yang Lu, Yaming Li, Rong Zhang, Kun Jin, Chunying Duan, Tetrahedron, 69, 9422(2013); (f) H. A. Muathen, Molecules, 8, 593(2003). (g) S. Samajdar, F. F. Becker, B. K. Banik, Tetrahedran Lett., 41, 8017(2000).

24. (a) N. Najmeh, A. M. Mehranpour, B. Elham, S. Zohre, Tetrahedron Lett., 53, 4841(2012); (b) G. K. S. Prakash, C. Panja, T. Mathew, V. Surampudi, N. A. Petasis, G. A. Olah, Org. Lett., 6, 2205(2004); (c) N. Iranpoor, H. Firouzabadi, N. Nowrouzi, D. Firouzabadi, Tetrahedron Lett., 47, 6879(2006); (d) N. Nowrouzi and Z. J. Mohammad, Tetrahedron Lett., 52, 5081(2011).

25. M. F. A. Dove, B. Manz, J. Montgomery, G. Pattenden, S. A. Wood, J. Chem. Soc., Perkin Trans., 1, 1589(1998).

26. A. K. Bose, S. N. Ganguly, M. S. Manhas, Sheetal Rao, S. Jeffrey, Uri. Pekelnya, E. P. Villars, Tetrahedron Lett., 47, 1885(2006).

27. (a) R. Rajagopal and K. V. Srinivasan, Synth. Commun., 33, 961(2003); (b) A. Cornelis, P. Laszlo, P. Pennetreau, J. Org. Chem., 48, 4771(1983); (c) T. Taniguchi and H. Ishibashi, Org. Lett., 12, 124(2010); (d) T.Taniguchi, T. Fujii, H. Ishibashi, J. Org. Chem., 75, 8126(2010); (e) T. Taniguchi, T. Fujii, H. Ishibashi, Org. Biomol. Chem., 9, 653(2011); (f) T. Naveen, S. Maity, U. Sharma, D. Maiti, J. Org. Chem., 78, 5949(2013).

28. (a) M. A.Zolfigol, E. Ghaemi, E. Madrakian, Synth. Commun., 30, 1689(2000); (b) M. S. Hosseini, M. Tavakolian, S. Ashenagar, Iranian J. Scienc. Tech., Transaction A, 34( A3) (2010).

29. (a) M. A. Zolfigol, E. Ghaemi, E. Madrakian, Molecules, 6, 614(2001); (b) M. A. Zolfigol, E. Madrakian, E. Ghaemi, Molecules 7, 734(2002); (c) M. A. Zolfigol, E. Ghaemi, E. Madrakian, Synlett., 191(2003).

30. B. Gigantee, A. O. Prazeres, M. J. Marcelo-Curto, A. Cornelis, P. Laszlo, J. Org. Chem., 60, 3445(1995).

31. V. Anuradha, P. V. Srinivas, P. Aparna, J. M. Rao, Tetrahedron Lett., 47, 4933(2006). 
32. J. M. Mellor, S. Mittoo, Parkes, Rachel, R. W. Millar, Tetrahedron. 56, 8019(2000).

33. D. M. Badgujar, M. B. Talwar, S. N. Asthana, P.P. Madhulikar, J. Sci. Ind. Res., 66, 250(2007).

34. M. M. Heravi, T. Benmorad, K. Bakhtiari, H. H. Oskooie, Y. S. Beheshtiha, G.U. J. Science., 22, 169-173(2009)

35. M. R. Heal, M. A. J. Harrison, J. N. Cape, Atmospheric Environment, 41, 3515(2007).

36. A. R. Pourali and G. Arezou, J. Chem. Sci., 63, 123 (2011).

37. Dai, Rong-Ji, Huang, Hui, Chen, Jie, Deng, Yu-Lin Xiao, Sheng-Yuan, Chinese J. Chem., 25, 1690(2007).

38. M. M. Heravi, H. A. Oskooie, B. Baghernejad, J. Chin. Chem. Soc., 54, 767(2007).

39. Bita Baghernejad., European J. Chem., 3, 125(2012)

40. (a) G. H. Barnett, H. J. Anderson, C. E. Loader, Can. J. Chem., 58, 409(1980); (b) R.Salmon, Encyclopedia of Reagents for Organic Synthesis, John Wiley \& Sons, New York: (2001).

41. I. Al'bina, Mikhaleva, V. Andrey, Ivanov, V. Elena, Skital'tseva, A. Igor', Ushakov, M.Alexander, Vasil'tsov, Boris A. Trofimov, Synthesis, 4, 587(2009).

42. S. A. Shackelford, M. B. Anderson, L. C. Christie, T. Goetzen, M. C. Guzman, M. A Hananel, W. D. Kornreich, H. Li, V. P. Pathak, A. K. Rabinovich, R. J. Rajapakse, L. K.Truesdale, S. M. Tsank, H. N. Vazir, J. Org. Chem., 68, 267(2003).

43. P. T. Anastas and J. C. Warner, Green Chemistry: Theory and Practice; Oxford University Press: New York, p. 30(1998).

44. A. S. Roger, A. Isabel, Ulf Hanefeld, Green Chemistry and Catalysis, First Edition Wiley-VCH, Weinheim, 2007, 1-47

45. M. M. Kirchhoff and P. T. Anastas, Acc. Chem. Res., 35, 686(2002).

46. G. R. Desiraju, B. S. Goud, "Reactivity of Solids. Present, Past, and Future," V. V. Boldyrev, Ed., Black-well Sciences, London, p. 223(1995).

47. (a) F. Toda, "Solid State Organic Reactions" Synlett., 5, 303(1993); (b) K. Tanaka, F. Toda, Chem. Rev., 100, 1025(2000).

48. (a) J. D. Lou and Z. N. Xu, Tetrahedron Letters, 43, 6095(2002); (b) S. Ramgopal, K. Ramesh, A. Chakradhar, N. Maasi Reddy, K.C. Rajanna, Tetrahedron Letters, 48, 4043(2007).

49. Y. Hemanth Sriram, K.C. Rajanna, M. Satish Kumar, M. Venkateswarlu, R.Madhusudan Raju, Rasayan J. Chem., 9, 769(2016).

50. R. S. Varma, D. E. Clark, W. H. Sutton, D. A. Lewis, "Microwaves: Theory and Application in Material Processing IV,” Eds., American Ceramic Society, Westerville, Ohio, p. 357(1997).

51. J. Hamelin, J. P. Bazureau, F. Texier-Boullet, A. Louby, "Microwave in Organic Synthesis," Wiley-VCH, Wein-heim, p. 253(2002).

52. Y.K. Srivastava, Rasayan J. Chem., 1, 888(2008),

53. J. Safari, S. Gandomi-Ravandi, M. Borjian Borujeni, J. Chem. Sci., 125, 1063(2013).

[RJC-1789/2017] 